\title{
Failed Supernovae Explain the Compact Remnant Mass Function
}

\author{
C. S. Kochanek ${ }^{1,2}$
}

\begin{abstract}
One explanation for the absence of higher mass red supergiants $\left(16.5 M_{\odot} \lesssim M \lesssim 25 M_{\odot}\right)$ as the progenitors of Type IIP supernovae ( $\mathrm{SNe}$ ) is that they die in failed SNe creating black holes. Simulations show that such failed SNe still eject their hydrogen envelopes in a weak transient, leaving a black hole with the mass of the star's helium core $\left(5-8 M_{\odot}\right)$. Here we show that this naturally explains the typical masses of observed black holes and the gap between neutron star and black hole masses without any fine-tuning of the SN mechanism beyond having it fail in a mass range where many progenitor models have density structures that make the explosions more likely to fail. There is no difficulty including this $\sim 20 \%$ population of failed SNe in any accounting of SN types over the progenitor mass function. And, other than patience, there is no observational barrier to either detecting these black hole formation events or limiting their rates to be well below this prediction.
\end{abstract}

Subject headings: stars: evolution - supergiants - supernovae:general -

\section{Introduction}

All massive $M \gtrsim 8 M_{\odot}$ stars undergo core collapse, but only some must explode as core collapse supernovae (ccSNe). All collapses leading to the formation of a neutron star must have a ccSNe to eject mass and avoid collapse to a black hole. Core collapse can lead to the formation of a black hole either through a failed $\mathrm{SN}$, where the stalled accretion shock never revives, or in a successful ccSN where sufficient mass falls back onto the proto-neutron star to cause collapse to a black hole. Little is observationally known about the balance between these scenarios. The diffuse supernova neutrino background sets an upper limit on the failed SN rate at roughly 50-75\% of the observed SN rate (Lien et al. 2010), and there is some evidence for a mismatch between massive star formation and SN rates, suggesting a significant failed SN rate (Horiuchi et al. 2011, but see Botticella et al. 2012), but theoretical studies generally favor low rates of failed $\mathrm{SNe}(\sim 10 \%$ of ccSN rate) at Solar metallicity (e.g. Woosley et al. 2002). We know nothing observationally about the formation of black holes in successful ccSNe, but it is a relatively common outcome in simulated explosions (e.g. Zhang et al. 2008, Fryer et al. 2012).

While not directly motivated by understanding the formation of black holes, surveys attempting to provide a census of the progenitor stars to successful SNe can reveal the existence of failed SNe. In particular, there appears to be a deficit of high mass progenitor stars compared to standard initial mass functions

\footnotetext{
${ }^{1}$ Department of Astronomy, The Ohio State University, 140 West 18th Avenue, Columbus OH 43210

${ }^{2}$ Center for Cosmology and AstroParticle Physics, The Ohio State University, 191 W. Woodruff Avenue, Columbus OH 43210
} 
(Kochanek et al. 2008, Smartt et al. 2009), This is best quantified for the deficit of higher mass $\left(\sim 20 M_{\odot}\right)$ red supergiant progenitors (Smartt et al. 2009), and it is interesting to note that this mass range also corresponds to stars with internal structures that make it more difficult for them to explode (e.g. O'Connor \& Ott 2011, Ugliano et al. 2012). Perhaps this deficit can be explained by observational biases such as stronger dusty winds around more massive stars (Walmswell \& Eldridge 2012, but see Kochanek et al. 2012), or by having stars in this mass range evolve away from being red supergiants before exploding (see the discussion in Smartt et al. 2009), but one simple explanation is that stars in the mass range $16.5 M_{\odot} \lesssim M \lesssim 25 M_{\odot}$ form black holes without a SN. The lower limit is set by the upper mass limit Smartt et al. (2009) found for Type IIP progenitors and $25 M_{\odot}$ is a reasonable estimate for the maximum mass of stars that undergo core collapse as red supergiants (see the discussion in Smartt et al. 2009). This mass range corresponds to $\sim 20 \%$ of core collapses.

In principle, neutrino (e.g. Abbasi et al. 2011, Alexeyev \& Alexeyeva 2002, Ikeda et al. 2007, or gravitational wave detection (e.g. Ott 2009) of a core collapse leading to black hole formation, combined with external astronomical observations of any resulting transient, would be the cleanest probe of this phenomenon. Unfortunately, such observations are only feasible in our Galaxy and its very nearest neighbors (e.g. Ando et al. 2005, Scholberg 2012), so the event rates are unpleasantly low if the failed SN rate is $\sim 20 \%$ of the SN rate. While any associated visible transient would be more easily observed in a nearby galaxy, almost all events in the Galaxy would be observable in the near-IR despite the high extinction in the Galactic plane (see Adams et al. 2013).

After pointing out the deficit of higher mass SN progenitors in Kochanek et al. (2008), we also outlined an approach to identifying black hole formation events without accompanying SN that was independent of the nature of any intervening transient. One carries out a "disappearance" experiment, monitoring a large number of evolved massive stars to see if any "vanish". We advocated this approach because the nature of the phenomenology that occurs between presence and absence was unclear and little studied. Some stars have density structures that would literally allow a direct collapse with almost no external signature Woosley \& Heger 2012), others might go through a phase where accretion onto the the black hole temporally and almost certainly unstably supports the remaining stellar envelope, or a weak shock might reach the surface of the star to create a weak explosive transient (e.g. Nadezhin 1980, Lovegrove \& Woosley 2013, Piro 2013). Our point in Kochanek et al. (2008) was that a search for black hole formation in failed SNe could be carried out independent of whether there was an associated, external transient because the initial and final states of the system were well defined. Moreover, this is the only direct detection method that is likely to succeed in the near term given supernova rates and the limited sensitivities of present and future neutrino and gravitational wave detectors.

The last available probe of these physical processes is the mass function of the resulting neutron stars and black holes. We show the observed masses of these remnants in Figure 1 using the summaries from Özel et al. (2010) and Özel et al. (2012). While this has many observational issues because we can only measure masses in binary systems, the observed mass function has three striking features (see, e.g., Bailyn et al. 1998, Özel et al. 2010, Farr et al. 2011, Kreidberg et al. 2012, Özel et al. 2012). First, neutron star masses have a narrow distribution. Second, there is a significant gap between the mass distribution of neutron stars 
and black holes. Third, while very high mass black holes exist, the typical black hole is far less massive than the stars believed to have created it. Özel et al. (2010) argue that selection effects due to the requirements for producing accreting binaries may bias the observed distributions against higher mass black holes $\left(M \gtrsim 10 M_{\odot}\right)$ but should not be producing a gap between neutron stars and black holes.

The simplest physical possibility would have been that successful SNe explosions lead to neutron stars with (roughly) the mass of the iron core (less neutrino losses etc.) and failed explosions lead to a black hole with the mass of the star at death. This would naturally lead to a gap in mass, with neutron stars having a mass $\simeq 1.4 M_{\odot}$ and black holes having the final stellar mass. The problem is that almost all stars at death are more massive than the typical mass of the observed black holes. In standard models (e.g. Zhang et al. 2008, Fryer et al. 2012), this forces black holes to be made in the course of successful ccSNe explosions so that mass can be ejected. The explosion is initiated in the core and the initial mass of the proto-neutron star is similar to an explosion in which a neutron star is formed, but there is then significant "fall back" of material onto the neutron star leading to the formation of a black hole. This allows formation of black holes less massive than their progenitor stars, but requires fine tuning of stellar mass loss and explosion energies. In particular, it is difficult to avoid a continuous distribution of remnant masses without the observed gap between neutron stars and black holes. These problems are illustrated in Figure 2, where we show the remnant mass distributions predicted by Zhang et al. (2008) and Fryer et al. (2012). We fully explain the construction of Figure 2 in §2. The Zhang et al. (2008) models have no gap, while the Fryer et al. (2012) models partially create one by changing the explosion energetics with progenitor mass (also see Belczynski et al. 2012). One practical difference between these models is that the Fryer et al. (2012) models were constructed in part to explain the remnant mass distribution while the Zhang et al. (2008) models were not. Perhaps the lack of similarity between the observed (Figure 1) and model (Figure 2) distributions is purely observational, but perhaps it is also evidence that fall back is not a good mechanism for explaining the masses of black holes.

In fact, simply accepting the evidence from progenitor studies that red supergiant stars with masses of $16.5 M_{\odot} \lesssim M \lesssim 25 M_{\odot}$ suffer failed supernova explosions and form black holes provides a new and very natural explanation for both the existence of the gap and the typical masses of black holes. The key is the observation by Nadezhin (1980) that the envelopes of red supergiants are so weakly bound that the weakening of the gravitational potential created by the mass lost in neutrinos during core collapse is sufficient to unbind the hydrogen envelope of the star. Lovegrove \& Woosley (2013) carried out detailed radiation-hydrodynamic simulations of this mechanism for $15 M_{\odot}$ and $25 M_{\odot}$ red supergiants, finding that the adjustment of the envelope to the neutrino mass loss can trigger a weak shock that unbinds the envelope as Nadezhin (1980) predicted. The result is a low luminosity $\left(\sim 10^{6} L_{\odot}\right)$, cool $(\simeq 3000 \mathrm{~K})$ transient lasting roughly one year and largely powered by the recombination energy of the envelope. Piro (2013) notes that there is also a shock break out pulse which is 10-30 times brighter, hotter $\left(\simeq 10^{4} \mathrm{~K}\right)$ and lasting roughly a week.

The key point for the remnant mass distribution is that the natural mass scale of the resulting black hole is the mass of the helium core of the progenitor star, thereby reproducing both the gap between neutron star and black hole masses and the characteristic minimum masses of black holes with no need for fine 
tuning stellar mass loss, the explosion mechanism, the amount of fall back, or binary evolution. In $\S 2$ we show some simple models of mass functions based on this idea and how to fit a significant population of failed SNe into the overall accounting for the deaths of massive stars. In $\S 3$ we discuss some additional implications and strategies for identifying these events.

\section{Results}

We generated Figure 2 using the following assumptions. We drew the progenitor masses from a Salpeter IMF over the mass range $8.5 M_{\odot}<M<100 M_{\odot}$, although the upper mass limit is quantitatively unimportant. We then assigned masses either by interpolating over the Zhang et al. (2008) models or using the analytic approximations in Fryer et al. (2012). We used the Solar metallicity SA model from Zhang et al. (2008), corresponding to an energy of 1.2 Bethe and a piston located at a fixed entropy per particle of $S / k=4$. Above and below the tabulated range from 12 to $100 M_{\odot}$ we simply used the results for the appropriate limiting mass. For the Fryer et al. (2012) models we assigned $1.4 M_{\odot}$ remnant masses to progenitors with $M<11 M_{\odot}$. As noted in $\S 1$, the results predict a continuum of black hole and neutron star masses and lack a clear peak in the observed mass range of black holes. When comparing Figures 1 and 2 one should not be comparing the relative numbers of black holes and neutron stars, but only the two classes separately. In Peicha et al. (2012) we modeled the masses of binary neutron stars from Özel et al. (2012) and found that they strongly disfavored models in which there was any fall back mass, while all the models used in Figure 2 must include fall back because it is the only way they can produce any low mass black holes.

In the Nadezhin (1980) mechanism, as confirmed by the simulations of Lovegrove \& Woosley (2013), the remnant mass from the failed SN of a red supergiant is set by the mass of the helium core of the star. There may be some hydrogen fall back contribution, but in the Lovegrove \& Woosley (2013) simulations it is small. Figure 3 shows the pre-SN mass, helium core mass, and the mass of the $Y_{e}$ core for the pre-SN stellar models of Woosley et al. (2002). The $Y_{e}$ core mass is a good proxy for neutron star masses in the absence of fall back and is defined by the point in the core where there is a significant jump in the electron abundance $Y_{e}$. In Pejcha et al. (2012) we found that the mass of the $Y_{e}$ core with no fall back was one of the better models for the mass distribution of binary neutron stars. In this model sequence, mass loss becomes increasingly important above $20 M_{\odot}$. Stars in the mass range corresponding to the missing red supergiant progenitors have helium core masses of between $5 M_{\odot}$ and $8 M_{\odot}$, almost exactly corresponding to the observed mass range of black holes.

We can now examine the remnant mass distribution if the more massive red supergiants undergo failed SN but eject their hydrogen envelopes based on the Nadezhin (1980) mechanism. Figure 4 shows the remnant mass distributions after we replace the remnant masses from the underlying models for the mass range $16.5 M_{\odot}<M<25 M_{\odot}$ with the helium core mass shown in Figure 3 . For all three cases, there is now a far more distinct peak at the observed masses of black holes, and a greatly reduced production of unobserved low mass black holes. The effect is most dramatic for the Zhang et al. (2008) model where the fraction of black holes (remnant masses $>2 M_{\odot}$ ) with masses between 5 and $10 M_{\odot}$ rises from $6 \%$ to $46 \%$. Even for the 
Fryer et al. (2012) models, where the parameters were in part tuned to better reproduce the mass function of binary black holes, the fractions rise from $\sim 40 \%$ to $\sim 70 \%$.

Smith et al. (2011) argue that adding such a population of failed $\mathrm{SNe}$ is difficult to reconcile with attempts to distribute SN types over the IMF. The essence of the argument is that the Type IIP fraction of $(48 \pm 6) \%$ found by Li et al. (2011) is so low that the proposed failed SN mass range of $16.5 M_{\odot} \lesssim M \lesssim$ $25 M_{\odot}$ needs to produce non-Type IIP SNe in order to match the observed SN type fractions. For example, in the absence of binaries, assigning the mass range from $M_{0}=9.4 M_{\odot}$ to $M_{1}=15.3 M_{\odot}$ to producing Type IIP $\mathrm{SNe}$, and $M>M_{1}$ to producing non-Type IIP SNe has a 48\% Type IIP fraction and is consistent $\left(\chi^{2}=1.5\right.$ for 1 dof) with the estimates of $M_{0}=8.5_{-1.5}^{+1.0} M_{\odot}$ and $M_{1}=(16.5 \pm 1.5) M_{\odot}$ by Smartt et al. (2009). However, this leaves no room to allot a mass range containing $\sim 20 \%$ of progenitors to failed $\mathrm{SNe}$.

Rather than being evidence against failed $\mathrm{SNe}$, this is really evidence for the importance of binaries, which was discussed by Smith et al. (2011) in other contexts. Since we lack a fully quantitative understanding of either mass loss by individual stars or mass transfer in binaries, we only consider three classes of objects. There are Type IIP SN, which we attribute to lower mass stars $\left(M_{0} \simeq 8.5 M_{\odot}<M<M_{1} \simeq 16.5 M_{\odot}\right)$. These limits are chosen to match the mass range found to be associated with Type IIP SNe by $\mathbf{S m a r t t}$ et al. (2009). Non-IIP SN are a combination of high mass stars $\left(M>M_{2} \simeq 25 M_{\odot}\right)$ that have lost mass due to either stellar evolution or binary mass transfer and a fraction $b$ of the IIP mass range where binary interactions lead to a non-IIP SN. The mass scale $M_{2} \simeq 25 M_{\odot}$ is roughly the highest mass at which stars both undergo core collapse as red supergiants and the range of progenitor masses which can be more difficult to explode (e.g. O'Connor \& Ott 2011 and Ugliano et al. 2012). Finally, the mass range $M_{1}<M<M_{2}$ leads to a failed SN. The interacting binary fraction is expected to be very high. For example, Sana et al. (2012) estimate that for O stars at birth, roughly $30 \%$ are effectively single, $24 \%$ merge, 33\% undergo some envelope stripping and $14 \%$ have some accretion, which means it is perfectly plausible that $b=30-50 \%$ of stars in the IIP mass range become non-IIP SNe due to interactions. In the IIP mass range, the donor star explodes as a non-IIP SN because of the mass transfer, and the recipient can explode as a non-IIP SN because it now evolves as a more massive star with greater mass loss. We discuss the potential effects of this binary fraction on the phenomenology of the failed $\mathrm{SNe}$ in $\S 3$.

Under these assumptions, we can simply solve for the binary fraction required to leave a $48 \%$ Type IIP fraction for successful SNe. For $M_{0}=8.5 M_{\odot}, M_{1}=16.5 M_{\odot}$ and $M_{2}=25 M_{\odot}$ this results in a binary fraction of $b=0.33$ which is quite reasonable. Raising the upper mass limit for failed SNe to $M_{2}=30 M_{\odot}$ only requires raising the binary fraction to $b=0.37$. For $M_{2}=25 M_{\odot}$ and the higher Type IIP fraction of 59\% found by Smartt et al. (2009) the binary fraction need only be $b=0.18$. Given all the other uncertainties, the main point is simply that given a reasonable fraction of binary-induced SN type transformations (from IIP to not IIP), there is no difficulty accommodating a significant rate of failed SNe in an accounting of SN types over the initial mass function. Figure 5 illustrates this graphically following the similar figures by Smith et al. (2011). 


\section{Discussion}

Assuming that high mass red supergiants die as failed SNe naturally solves two observational puzzles: (1) the failure to find progenitors in this mass range (e.g. Kochanek et al. 2008, Smartt et al. 2009), and (2) the peculiar mass distribution of compact remnants (e.g., Bailyn et al. 1998, Özel et al. 2010, Farr et al. 2011, Özel et al. 2012). While we lack a fully predictive theory of core collapse events, it is also true that many stellar models in this mass range have density structures that render them more difficult to explode (e.g. O’Connor \& Ott 2011, Ugliano et al. 2012).

Models of successful SNe can form black holes with masses of 5 to $10 M_{\odot}$ by carefully tuning the stellar mass loss and the explosion energetics to achieve the correct amount of mass fall back. We used the examples of Zhang et al. (2008), which poorly matches the observed black hole mass distribution, and Fryer et al. (2012), which does better. However, if we simply excise the mass range from $16.5 M_{\odot}$ to $25 M_{\odot}$ from these models and instead make them failed SNe in which the hydrogen envelope is ejected by the Nadezhin (1980) mechanism, then we naturally create large numbers of black holes in the observed mass range. The mass scale comes naturally from the mechanism because only the weakly bound hydrogen envelope can be ejected. The black hole mass scale is simply the mass of the helium cores of the stars ending their lives as failed SNe. Since there is no longer any need to tune the amount of fall back mass to produce low mass black holes in successful SNe, we could now allow all successful SNe to form neutron stars with no fall back, which would eliminate the residual problem that other progenitor mass ranges also produce low mass black holes in the Zhang et al. (2008) or Frver et al. (2012) models. Having no fall back would also better match the neutron star mass distribution (Pejcha et al. 2012). Similarly, one could add a population of failed SN associated with more compact, higher mass stars where the final mass would be that of the star at the time of explosion. The key point for models of the remnant mass distribution is that the Nadezhin (1980) mechanism provides a means of producing $\sim 7 M_{\odot}$ black holes that does not depend on either mass loss or the fall back mechanism.

If we build a simple model where stars from $8.5 M_{\odot}$ to $16.5 M_{\odot}$ produce Type IIP SNe unless modified by binary interactions, stars from $16.5 M_{\odot}$ to $25 M_{\odot}$ produce failed $\mathrm{SNe}$, and higher mass stars produce non-IIP SNe, then it is easy to match the Li et al. (2011) SN type fractions if roughly $1 / 3$ of potential IIP progenitors are modified by binary interactions and become non-IIP SNe. In such a model, $18 \%$ of core collapses fail, corresponding to a failed SN rate that is $f=22 \%$ of the $\mathrm{SN}$ rate. Using the higher IIP fraction found by Smartt et al. (2009) allows a lower binary modification fraction. Invoking such a fraction of binary interactions is consistent with the estimates of Sana et al. (2012) from the statistics of O star binaries.

If we translate the luminosities and temperatures estimated by Lovegrove \& Woosley (2013) and Piro (2013) into magnitudes assuming black body spectra, the absolute visual magnitude of the transient and the shock break out pulses are roughly $M_{V} \simeq-8.7 \mathrm{mag}\left(M_{I} \simeq-11.2\right)$ and $M_{V} \simeq-13.5 \mathrm{mag}\left(M_{I} \simeq-13.7\right)$, respectively (Adams et al. 2013). The transient is significantly brighter at longer wavelengths because of its low temperature. If we assume that the typical Type IIP magnitude is $M_{I I P}=-16$ mag, then a field survey finding $N_{I I P}$ Type IIP SNe should find

$$
N_{\text {Ned }}=f N_{I I P} 10^{0.6\left(M_{I I P}-M_{N e d}\right)}
$$


Nadezhin (1980) events, where the magnitude dependence is created by the (Euclidean) dependence of the survey volume on transient luminosity. For the extended, cool transient, the expected numbers are very low, $\sim 10^{-3}$ to $10^{-4} f N_{I I P}$ depending on the survey band pass. This phase of the transient also does not stand out significantly from other slow variations in high luminosity stars. Hence, as suggested by Piro (2013), normal SN surveys should focus on the shock break out pulse from these events where the expected number would be $\sim 0.03 f N_{I I P}$. Such a search will require cadences closer to daily than weekly in order to sample the transient well-enough to have confidence in the detection and to motivate a search for the longer duration, fainter transient with larger telescopes. Fortunately, the break out peak and duration appear to occupy a region of transient space without significant, known backgrounds, as they should be significantly more luminous than classical novae of the same duration. Achieving a $90 \%$ confidence limit of $f<0.1$ requires a survey where the expectation value is 2.3 events, so surveys containing $10^{3}$ Type IIP SNe that could have detected the break out peaks of these transients at high efficiency will begin to provide strong constraints on the existence of this mechanism. As presently designed, however, most field surveys for SN have cadences that will make it difficult to achieve a high efficiency for detection of these transients since they will sample the events poorly (e.g. cadences of 5, 7 and 3 days for PTF, Pan-STARRS1 and LSST, respectively, see Rau et al. (2009) for a summary of surveys).

A targeted survey focused on nearby galaxies, such as our more general search for failed supernovae (Kochanek et al. 2008), has little prospect of detecting the break out pulse because of its low cadence (> monthly), but would have no difficulty following the longer transient since it was already designed to search for the disappearance of far less luminous stars. Here the rate is limited simply by the rarity of the SNe, since $N_{I I P} \simeq 1$ /year for local galaxies, leading to an expected rate of $\simeq f /$ year for this class of failed supernovae. Thus, achieving a 90\% confidence limit that $f<0.1$ requires two decades of monitoring nearby galaxies which is painfully long but entirely feasible. Of course if $f \simeq 0.2$, the probability of finding such an event in a decade is quite high (86\%) and it is only for these nearby events that we are guaranteed to be able to convincingly say that the progenitor star has vanished.

Finally, we should not expect all these events to have the luminous counterparts predicted by Lovegrove \& Woosley (2013). First, as noted by Lovegrove \& Woosley (2013), the neutrino mass loss may not be large enough to trigger envelope ejection in all cases. Second, like the stars which eventually have successful SNe, a significant fraction of the stars that will become failed SNe will be in binaries and will have part or all of their hydrogen envelopes stripped before death. To the extent that the interior structure that will lead to a failed SNe is not significantly altered by the mass loss, these stars will still end as failed SNe. If there is remaining hydrogen and it is still in an extended, low binding energy envelope, then we would still expect a transient associated with core collapse but it would be weaker. In some senses, these would be the failed $\mathrm{SN}$ equivalents of Type IIL or IIb SNe. If the mass loss leads the envelope to collapse or if all the hydrogen is stripped, then there would likely be no luminous transient. Since we are unable to predict the outcome of core collapse from first principles, it is difficult to address these scenarios quantitatively. However, if $\sim 1 / 3$ of stars which would otherwise become IIP SNe do not do so because of binary interactions, we might expect a similar fraction of failed $\mathrm{SNe}$ to be modified. 
I would like to thank J. Beacom, A. Gould, T. Piro, K. Stanek and T. Thompson for discussions or comments and F. Özel for supplying the data used in Figure 1

\section{REFERENCES}

Abbasi, R., Abdou, Y., Abu-Zayyad, T., et al. 2011, A\&A, 535, A109

Adams, S. M., Kochanek, C. S., Beacom, J. F., Vagins, M. R., \& Stanek, K. Z. 2013, arXiv:1306.0559

Alexeyev, E. N., \& Alexeyeva, L. N. 2002, Soviet Journal of Experimental and Theoretical Physics, 95, 5

Ando, S., Beacom, J. F., Yuksel, H. 2005, Physical Review Letters, 95, 171101

Bailyn, C. D., Jain, R. K., Coppi, P., \& Orosz, J. A. 1998, ApJ, 499, 367

Belczynski, K., Wiktorowicz, G., Fryer, C. L., Holz, D. E., \& Kalogera, V. 2012, ApJ, 757, 91

Botticella, M. T., Smartt, S. J., Kennicutt, R. C., et al. 2012, A\&A, 537, A132

Farr, W. M., Sravan, N., Cantrell, A., et al. 2011, ApJ, 741, 103

Fryer, C. L., Belczynski, K., Wiktorowicz, G., et al. 2012, ApJ, 749, 91

Gerke, J., et al., in preparation

Horiuchi, S., Beacom, J. F., Kochanek, C. S., et al. 2011, ApJ, 738, 154

Ikeda, M., Takeda, A., Fukuda, Y., et al. 2007, ApJ, 669, 519

Jennings, Z. G., Williams, B. F., Murphy, J. W., et al. 2012, ApJ, 761, 26

Kochanek, C. S., Beacom, J. F., Kistler, M. D., et al. 2008, ApJ, 684, 1336

Kochanek, C. S., Khan, R., \& Dai, X. 2012, ApJ, 759, 20

Kreidberg, L., Bailyn, C. D., Farr, W. M., \& Kalogera, V. 2012, ApJ, 757, 36

Li, W., Leaman, J., Chornock, R., et al. 2011, MNRAS, 412, 1441

Lien, A., Fields, B. D., \& Beacom, J. F. 2010, Phys. Rev. D, 81, 083001

Lovegrove, E., \& Woosley, S. E. 2013, ApJ, 769, 109

Nadezhin, D. K. 1980, Ap\&SS, 69, 115

O'Connor, E., \& Ott, C. D. 2011, ApJ, 730, 70

Ott, C. D 2009, Classical and Quantum Gravity, 26, 063001 
Özel, F., Psaltis, D., Narayan, R., \& McClintock, J. E. 2010, ApJ, 725, 1918

Özel, F., Psaltis, D., Narayan, R., \& Santos Villarreal, A. 2012, ApJ, 757, 55

Pejcha, O., Thompson, T. A., \& Kochanek, C. S. 2012, MNRAS, 424, 1570

Piro, A. L. 2013, ApJ, 768, L14

Rau, A., Kulkarni, S. R., Law, N. M., et al. 2009, PASP, 121, 1334

Sana, H., de Mink, S. E., de Koter, A., et al. 2012, Science, 337, 444

Scholberg, K. 2012, Annual Review of Nuclear and Particle Science, 62, 81

Smartt, S. J., Eldridge, J. J., Crockett, R. M., \& Maund, J. R. 2009, MNRAS, 395, 1409

Smith, N., Li, W., Filippenko, A. V., \& Chornock, R. 2011, MNRAS, 412, 1522

Timmes, F. X., Woosley, S. E., \& Weaver, T. A. 1996, ApJ, 457, 834

Ugliano, M., Janka, H.-T., Marek, A., \& Arcones, A. 2012, ApJ, 757, 69

Walmswell, J. J., \& Eldridge, J. J. 2012, MNRAS, 419, 2054

Woosley, S. E., Heger, A., \& Weaver, T. A. 2002, Reviews of Modern Physics, 74, 1015

Woosley, S. E., \& Heger, A. 2012, ApJ, 752, 32

Zhang, W., Woosley, S. E., \& Heger, A. 2008, ApJ, 679, 639 


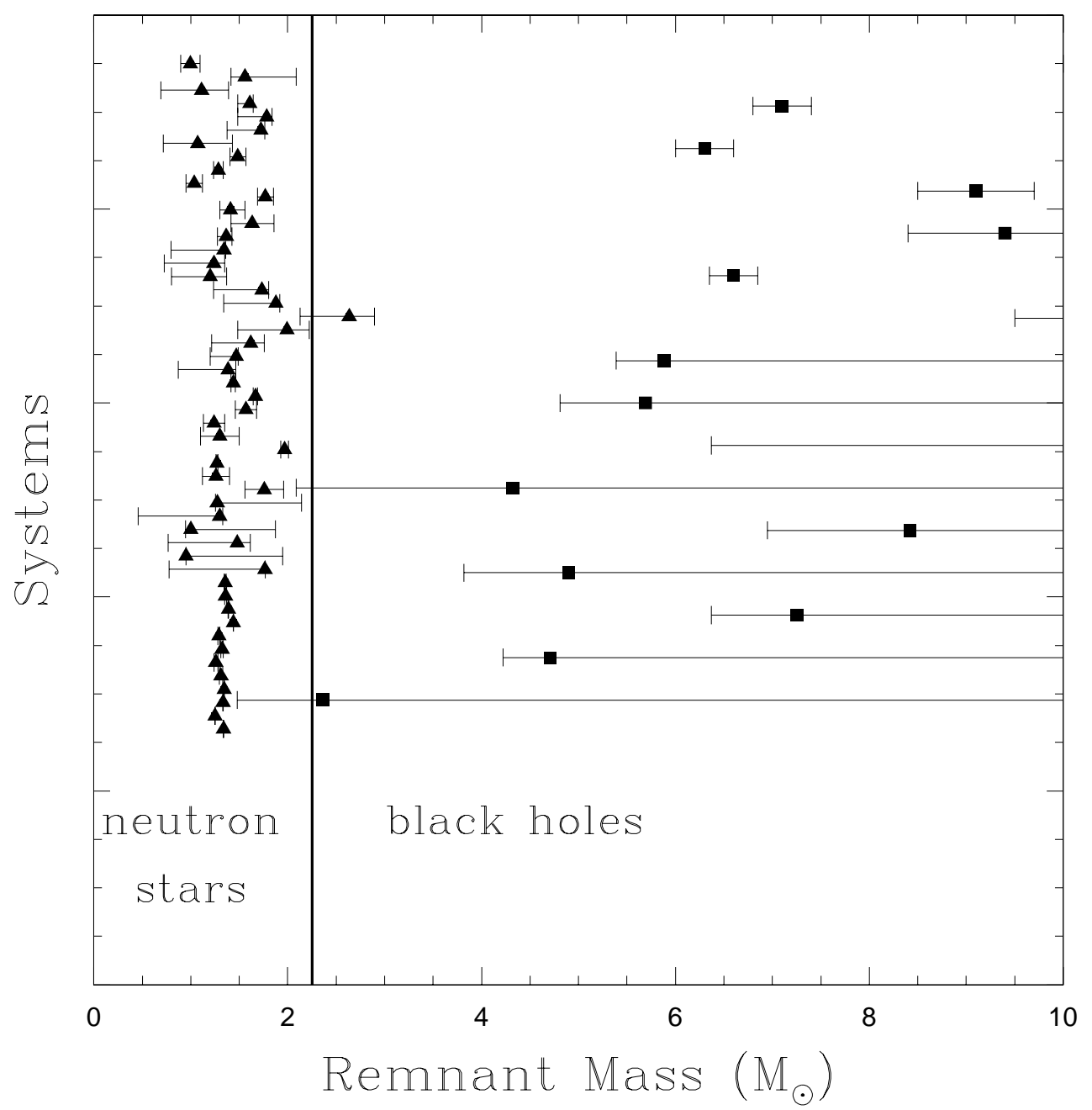

Fig. 1.- Observed masses of neutron stars (filled triangles) and black holes (filled squares) from Özel et al. (2010) and Özel et al. (2012). The thick solid line at 2.25M $\odot$ roughly marks the maximum mass of a neutron star. The relative numbers of neutron stars and black holes cannot be quantitatively compared. 


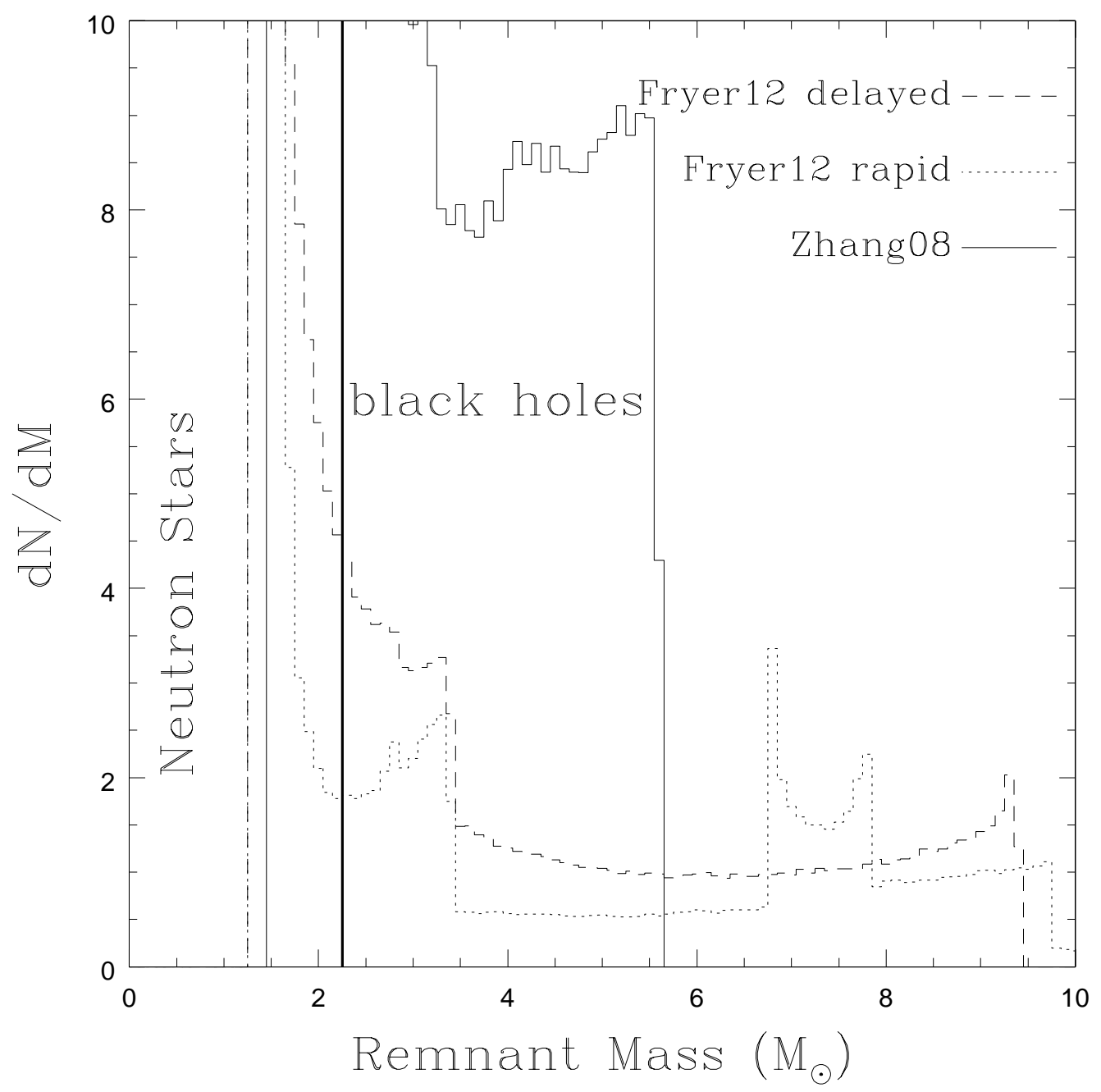

Fig. 2. - Remnant mass distributions for the explosion models of Zhang et al. (2008) (solid) and Fryer et al. (2012), where dotted lines show the distribution for rapid explosions and dashed lines show the distribution for delayed explosions. The distributions are normalized by the average number of remnants between $5 M_{\odot}$ and $10 M_{\odot}$. The models predict distributions dominated by low mass $\left(<5 M_{\odot}\right)$ black holes and lack any clear gap between the masses of neutron stars and black holes. The thick solid line at $2.25 M_{\odot}$ roughly marks the maximum mass of a neutron star. 


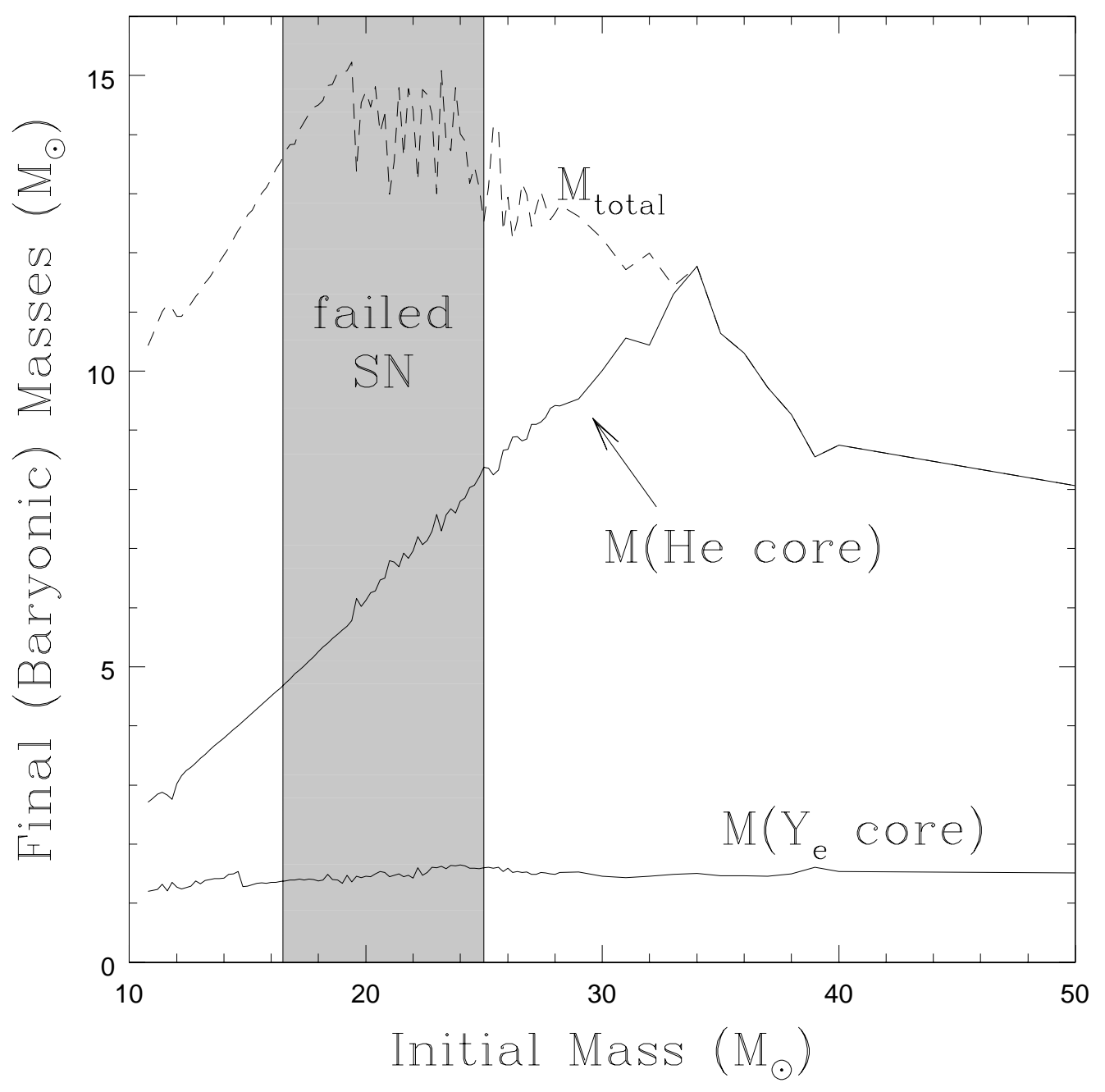

Fig. 3.- Pre-supernova structure of massive stars from Woosley et al. (2002). The dashed line shows the final mass of the star. The solid lines show the mass of the $Y_{e}$ core, which will roughly correspond to the mass of any resulting neutron star, and the mass of the helium core. Note the enormous mass loss associated with the higher mass stars. The shaded region encompass the mass range from $16.5 M_{\odot}$ to $25 M_{\odot}$ that we associate with failed supernovae. 


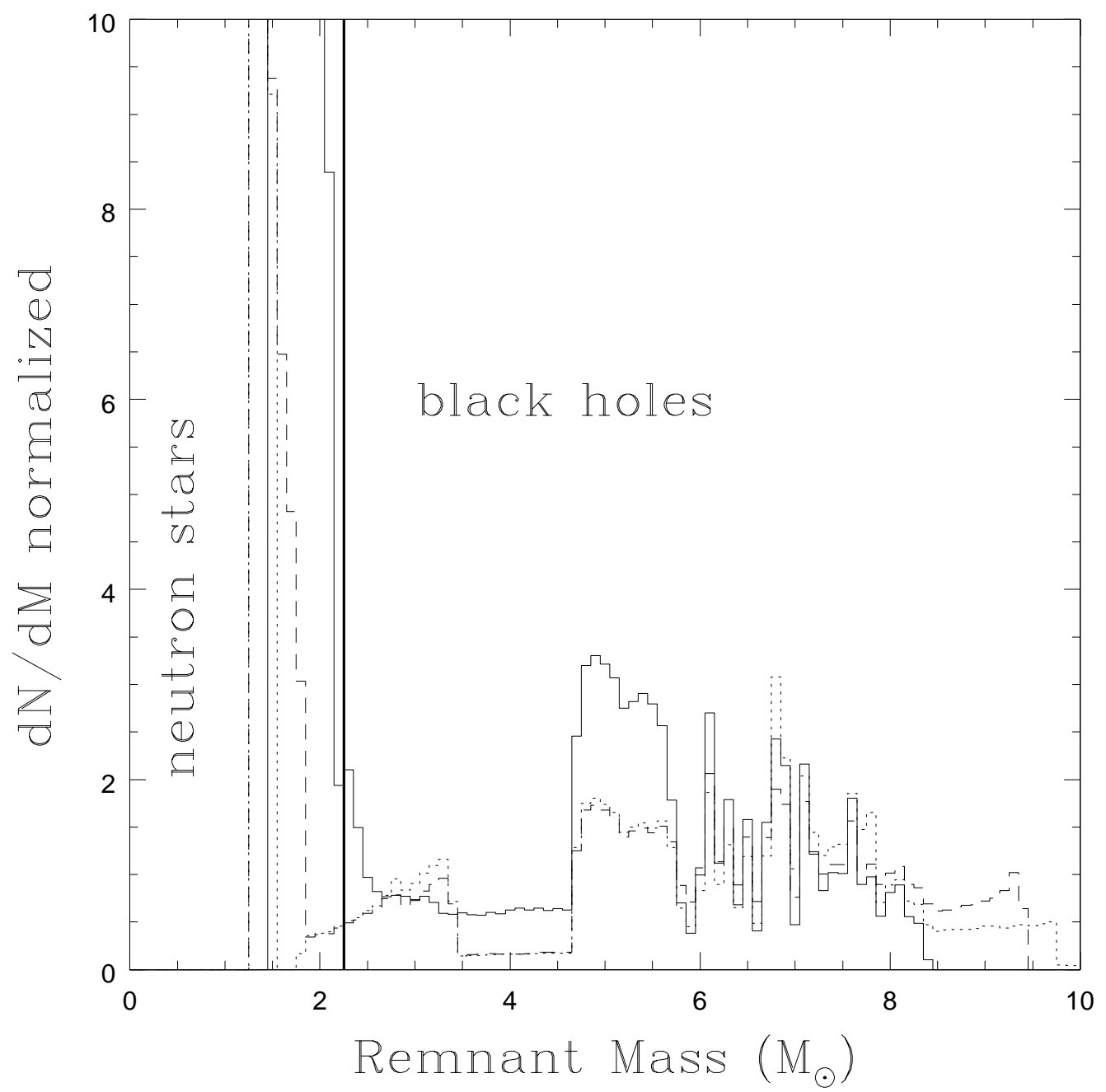

Fig. 4.- Remnant mass distributions if the core collapse of stars in the mass range from $16.5 M_{\odot}$ to $25 M_{\odot}$ leads to the formation of black holes with the helium core mass from the models of Wooslev et al. (2002) (Figure 3). Outside this mass range we continue to use the results from Zhang et al. (2008) (solid) and Fryer et al. (2012), where dotted lines show the distribution for rapid explosions and dashed lines show the distribution for delayed explosions. The distributions are normalized by the average number of remnants between $5 M_{\odot}$ and $10 M_{\odot}$. The black hole mass distributions now have a distinct peak in the observed mass range and far fewer low mass black holes. These low mass black holes could be completely eliminated by adopting explosion models with no fall back. The thick solid line at $2.25 M_{\odot}$ roughly marks the maximum mass of a neutron star. 


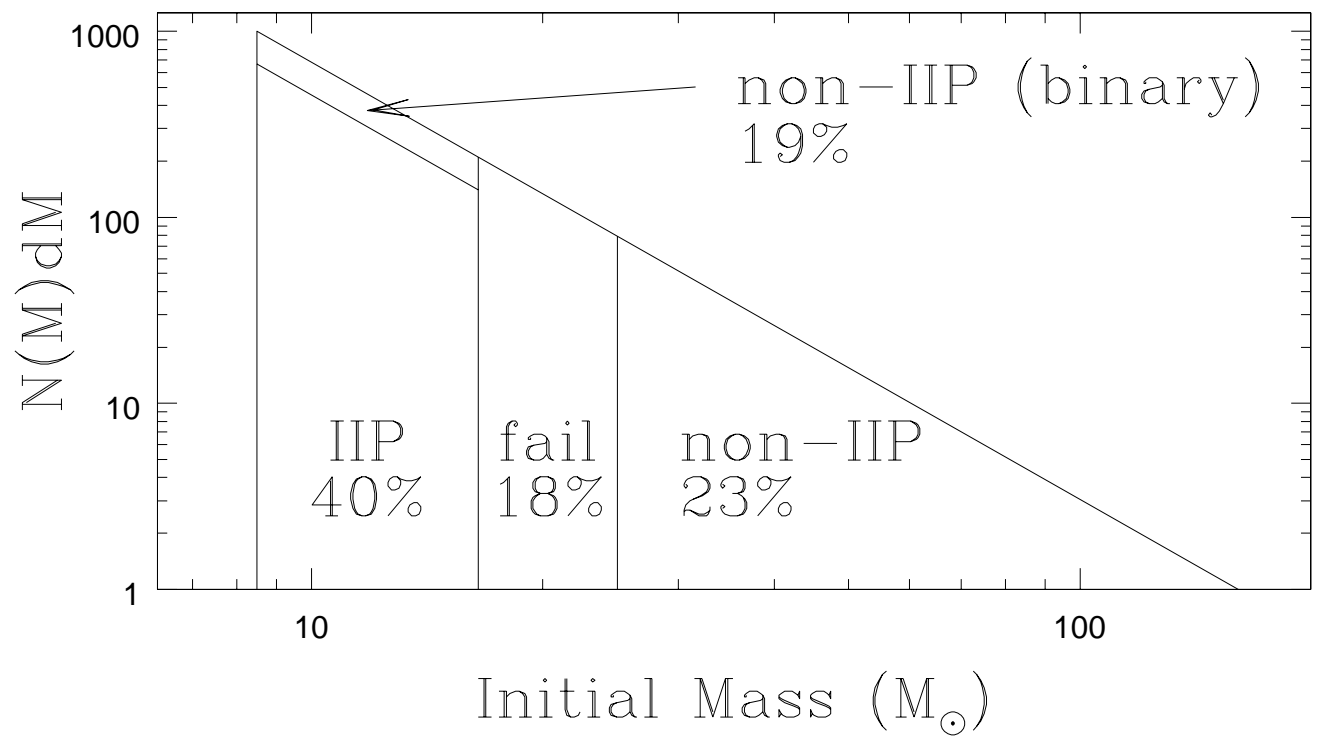

Fig. 5.- Mass ranges implied for SN types following Smith et al. (2011) but including a failed SN component. The fractions represent the fraction of all core collapses, so while the fraction of core collapses leading to Type IIP (non-IIP) SNe is 40\% (42\%), the fraction of SNe that are Type IIP (non-IIP) is $48 \%$ (52\%). Similarly, $18 \%$ of core collapses fail, but the failed SN rate is $22 \%$ of the SN rate. The mass loss making the high mass stars have non-IIP SNe can be an arbitrary mixture of binary and single star processes. The interacting binary fraction for this model is $b=33 \%$, and we discuss how this may affect the phenomenology of the failed SNe in $\S 3$. 